\title{
EVALUACIÓN EXPERIMENTAL DE UN MODELO DE PROGRAMACIÓN LINEAL PARA EL PROBLEMA DE RUTEO DE VEHÍCULOS (VRP)
}

\author{
Juan Manuel Machuca de Pina \\ jmachuca@ulima.edu.pe \\ Universidad de Lima. Lima, Perú \\ Michael Dorin \\ mike.dorin@stthomas.edu \\ University of St. Thomas, Minnesota. Estados Unidos \\ Alicia Isabel García Yi \\ agarciay@ulima.edu.pe \\ Universidad de Lima. Lima, Perú
}

\section{Resumen}

El presente artículo tiene como objetivo proponer un criterio cuantitativo para evaluar la viabilidad de implementar soluciones basadas en programación lineal para resolver el problema de ruteo de vehículos (VRP). Se utilizó un diseño experimental para medir el tiempo relativo de solución con un modelo de programación lineal propuesto. La muestra utilizada fue aleatoria utilizando tres escenarios de dispersión de puntos de entrega: poco dispersos, dispersos y muy dispersos. Se utilizó un solver de programación lineal con el objetivo de determinar el tiempo y las iteraciones necesarias para encontrar la solución del modelo. Como resultado se encontró el tiempo de solución en función de la cantidad de puntos de entrega, la cantidad de iteraciones para los escenarios propuestos y se pronostica el tiempo necesario para resolver el problema utilizando el modelo propuesto. Se concluye con una propuesta de cantidad de puntos viables para ser resueltos por programación lineal.

Palabras clave: programación lineal, complejidad, ruteo, vehículos, puntos de entrega

\section{Abstract}

\section{Experimental evaluation of a linear programming model for solving the vehicle routing problem (VRP)}

This article aims to propose a quantitative criterion to evaluate the feasibility of implementing solutions based on linear programming for solving the vehicle routing problem (VRP). An experimental design was used to measure the relative solution time with a proposed linear programming model. The sample was randomized employing three dispersion scenarios of the delivery points: poorly scattered, scattered and very scattered. A linear programming solver was used to determine the time and iterations necessary for solving the model. As a result, the solution time was found in terms of the number of delivery points and the number of iterations for the proposed scenarios, and the time required to solve the problem was predicted using the proposed model. The research concludes with a proposal of the number of viable points to be solved by linear programming.

Keywords: linear programming, complexity, routing, vehicles, delivery points 


\section{Introducción}

Las empresas enfrentan diariamente el problema de diseñar rutas para el reparto vehicular de mercaderías de manera más eficiente y a menor costo. A pesar de que el desarrollo tecnológico actual ofrece a los usuarios soluciones de hardware y software potentes, la práctica actual generalizada se basa en el diseño manual de rutas a cargo del personal de logística u operaciones, más que en soluciones automatizadas soportadas por modelos matemáticos o heurísticos.

La ruta diseñada mediante labor manual es menos o más eficiente en la medida en que el trabajador adquiere experiencia en el puesto y, aún así, este diseño tiene un alto riesgo de error. Los modelos matemáticos de programación lineal son una alternativa de automatización del diseño. Sin embargo, estos modelos consumen un tiempo computacional elevado que puede superar el tiempo disponible para decidir la ruta e iniciar el reparto de mercancías. Esto genera una brecha perceptual en el diseño y la prestación del servicio computacional entre lo que los clientes esperaban y lo que perciben haber recibido del proveedor informático (Lovelock y Wirtz, 2009).

Los modelos matemáticos de programación lineal permiten la optimización restringida de los procesos empresariales. En el diseño de modelos de ruteo se pueden incluir diferentes criterios que influyen en el diseño de las rutas y en la percepción de calidad de estas soluciones. Tales variables pueden ser: el costo de transporte, el tiempo de entrega, la capacidad de carga de los vehículos, las distancias, entre otras. Sin embargo, a pesar de la evolución de los microprocesadores, estas soluciones pueden suponer tiempos demasiado elevados de procesamiento como para ser consideradas por el software de soporte de decisiones. En ese sentido Azzara (2010) indica que la investigación debería contribuir a mantener las transacciones eficientes y productivas.

Se conoce que en ciudades con condiciones de tránsito caótico como Lima, los habitantes pierden tiempo valorizado aproximadamente en el $25 \%$ de sus ingresos por los problemas de transporte de la ciudad ("Limeños pierden el $25 \%$ de sus ingresos por el tránsito vehicular", 2017). Además están los problemas para reducir el impacto del costo de los combustibles en el costo de transporte debido a la baja incidencia del uso de gas natural (Machuca y Taquía, 2009). Entre las empresas que requieren, para ser cada vez más competitivas, ofrecer servicios complementarios que añadan valor para sus clientes (Lovelock y Wirtz, 2009), destacan algunas, como las dedicadas al delivery de comida, que ofrecen atender pedidos en 30 minutos o menos, bajo la penalidad de no cobrar el pedido si llegan tarde, y otras como las dedicadas a la salud con atención médica a domicilio, que programan su itinerario de citas ofreciendo puntualidad en la atención. En los casos mencionados las pérdidas ocasionadas debido a dificultades en el transporte de los productos o a la entrega del servicio pueden ser significativas. 
Obtener la ruta óptima para los vehículos permite mejorar la satisfacción del cliente, cumplir con las políticas de calidad, optimizar los costos de transporte, mejorar la productividad de las unidades de transporte y del personal asignado a éstas. Estos son factores que influyen directa o indirectamente en la economía de la empresa.

El reparto de producto es parte del servicio al cliente, sin embargo, la segmentación clásica de mercados ha puesto poca atención al impacto que esta tiene en las operaciones de distribución, generando una verdadera desconexión entre la búsqueda de formas para comprender qué quieren los clientes y el mapeo de las rutas de transporte. Los clientes tienen un paquete de expectativas holístico que se manifiesta en una jerarquía de atributos que incluyen la velocidad y continuidad en el abastecimiento (Gattorna, 2009).

El reemplazo del trabajo manual implica, la mayoría de las veces, el uso de software para agilizar los procesos. Pero surge una discusión sobre la complejidad del software. Una forma de entender la complejidad está orientada a sopesar cuán difícil es entender el código fuente de un programa por humanos. Otro concepto de complejidad se refiere a qué tan laborioso es procesar un algoritmo para una computadora promedio. Aunque en general se aprecia la capacidad de las computadoras para manejar problemas demasiado intrincados para los humanos, en muchos casos las dos áreas de complejidad están conectadas. La teoría de la complejidad computacional se utiliza para clasificar los problemas algorítmicos en función de su dificultad. Cuanto más complejo es un algoritmo, más recursos computacionales se requieren para ejecutarlo en tiempos aceptables (Dantzig, 2002). Como se describe en este artículo, el uso de modelos de optimización matemática para encontrar soluciones puede poseer ambos tipos de complejidad de la teoría computacional, tanto para los humanos como para la máquina. La notación llamada Big O se usa para describir el nivel de complejidad computacional de un algoritmo. La notación Big O describe el peor camino de un algoritmo a medida que aumenta el tamaño de los datos (Cook, 1983). Este documento no analiza todos los aspectos de la medición de la complejidad computacional, pero se conoce que los algoritmos de optimización matemática se pueden describir comúnmente como que poseen complejidad cuadrática $\left(O\left(n^{2}\right)\right)$ o exponencial $\left(O\left(k^{n}\right)\right)$ en función del tiempo que requieren. En pocas palabras, esto significa que el número de variables puede cambiar en una cantidad pequeña mientras el tiempo y los recursos necesarios para calcular una solución pueden aumentar drásticamente. Aunque la teoría de la complejidad computacional se difunde a nivel mundial en las universidades, su utilidad se pasa por alto o se considera difícil de aplicar.

Como se mencionó anteriormente, una decisión crucial que la empresa debe tomar al aplicar los modelos de optimización matemática es si, con base en el tamaño de sus datos, es adecuado o no seleccionar el uso de modelos heurísticos por los costos que estos generan. Un proceso de optimización debe determinar una solución en un tiempo apropiado (Dantzig, 2002). Por ejemplo, usar un algoritmo de programación lineal para la optimización no es 
práctico si los recursos disponibles para el procesamiento informático no son suficientes para obtener una solución en un tiempo razonable. No tiene sentido usar un modelo de optimización, por ejemplo, para un proceso logístico, cuyo tiempo usado para el cálculo es mucho mayor que el tiempo que se ahorra en dicho proceso logístico con la solución obtenida.

\section{Revisión de literatura}

El diseño de rutas es conocido como el problema de ruteo de vehículos (vehicle routing problem, VRP). Las propuestas para resolver este problema han sido abordadas con modelos matemáticos, heurísticos y metaheurísticos aplicados al VRP y sus variantes (Arboleda-Zúñiga, López y Lozano, 2016).

El problema básico consiste en diseñar la ruta para una flota de vehículos idénticos, donde el vehículo inicia la ruta en un depósito o almacén y termina la ruta en el mismo punto de salida, seleccionando un camino, conformando un conjunto de arcos que pasan por los puntos de entrega de mercancías o bienes por los que necesariamente debe pasar el vehículo, asignándose peso o volumen de entrega sin sobrepasar la capacidad de carga (Laporte, 2009).

Las soluciones basadas en modelos matemáticos de programación lineal han sido propuestas desde hace más de 50 años, siendo la primera la de Dantzig y Ramser en 1959 (Golden, Wasil, Kelly y Chao, 1998; Laporte, 2009). La propuesta inicial ha evolucionado para resolver problemas con mayor cantidad de restricciones (Alvarez, Lerga, Serrano, y Faulin, 2017; Laporte, 2009) y también se ha especializado con propuestas de aplicaciones concentradas en la recolección de desperdicios y modelos multiobjetivo para el transporte de ayuda en crisis humanitarias ocasionadas por desastres (Han y Cueto, 2015; Tzeng, Cheng y Huang, 2007). Además se añadió el comportamiento de los conductores como uno de los factores a ser considerados para incluirse en el diseño de rutas (Alvarez, Lerga, Serrano y Faulin, 2017; Srivatsa Srinivas y Gajanand, 2017).

Diversas propuestas han abordado las diferentes limitaciones del problema original. Onut, Kamber y Altay (2014) realizaron una propuesta para minimizar el costo de transporte en flotas heterogéneas de vehículos, aplicándola a una flota de gas licuado de petróleo en Turquía para atender 13 estaciones y dos tipos de unidades de transporte. Se utilizó un modelo matemático que fue resuelto utilizando GAMS con datos reales de empresas del sector, encontrando solución al modelo propuesto en 15 minutos de procesamiento.

Alternativamente, el problema ha sido enfocado a través de heurística y metaheurística. Daza, Montoya y Narducci (2009) propusieron como solución al problema logístico de transporte en Colombia un modelo matemático basado en el algoritmo Greedy que fue probado en un problema logístico con 80 nodos. Los autores evaluaron la aplicación de metaheurística en dos fases para el enrutamiento en vehículos de capacidad limitada, encontrando que 
la metaheurística halló mejores soluciones que la heurística y que, con datos reales y ficticios, la solución obtenida para el enrutamiento con 15 camiones se obtiene en un tiempo computacional inferior a un minuto.

Desde el punto de vista de aplicabilidad en el desarrollo de Sistemas de Soporte de Decisiones (DSS), la aparición de tecnologías de información de uso cotidiano hace necesario evaluar la inclusión de Google Maps API en el diseño de rutas (Alvarez, Lerga, Serrano y Faulin, 2017).

\section{Metodología}

Se utilizó la teoría básica de complejidad computacional para estimar si un modelo matemático de optimización puede completarse en un periodo de tiempo razonable. En la experimentación se utilizó un modelo de programación lineal para el VRP, diseñado para programar un vehículo sin consideraciones de capacidad u otro tipo de limitaciones. Por inferencia se deduce que modelos con más variables y restricciones que las incluidas en el modelo de prueba, necesariamente consumirán un mayor tiempo de procesamiento en un computador promedio. Se hizo una caracterización del comportamiento del algoritmo de solución del modelo matemático con diversos conjuntos de datos para estimar la cantidad de tiempo requerido para usar un modelo de optimización.

Para comenzar este proceso, se ejecutó el algoritmo de optimización matemática evaluando individualmente cada elemento de múltiples conjuntos de datos. Cada conjunto estuvo caracterizado por poseer elementos con diferentes cantidades de puntos y el mismo atributo de dispersión que representaron a los escenarios propuestos. Para determinar el grado de dispersión de los puntos se utilizó el coeficiente de correlación de Pearson. En cada escenario el conteo de variables cambió para cada elemento debido al incremento de los puntos atendidos. A pesar de ello, se esperó encontrar valores de conteo que pertenezcan a un intervalo de tiempo razonable en el que se desea que se complete la ejecución del algoritmo. Cada vez que se ejecutó el algoritmo de optimización, se registró el número de variables, el tiempo para encontrar la solución y el número de iteraciones que empleó la herramienta de software para resolver el modelo de programación lineal (solver).

Para determinar si es viable realizar una optimización matemática, se calcula una estimación de la magnitud del tiempo empleado por la herramienta de software para encontrar la solución óptima. Esto se hizo realizando una proyección mediante una regresión lineal simple.

Se utilizó para la evaluación una adaptación del modelo propuesto por Baldacci, Hadjiconstantinou y Mingozzi (2004) para la programación del ruteo (VRP). La adaptación redujo la programación a un vehículo de capacidad limitada $C$, que debe entregar $n$ paquetes, cada uno en un destino diferente, para finalmente retornar al punto de partida inicial denominado punto cero (0) con una formulación de función objetivo que buscará establecer la ruta 
en la que la unidad de transporte realice el menor recorrido durante el reparto a $\mathrm{n}$ puntos de entrega. Se define $V=\langle 0,1,2,3, \ldots, n\rangle$ como el conjunto de $n$ puntos unidos por los arcos $(i, j)$ por los que puede pasar el vehículo, al partir desde el punto 0 . En esta formulación se define el conjunto $V$ aumentado como $V=V \cup\{n+1\}$ que se obtiene al duplicar el punto de partida como destino final. Se considera la distancia $D_{i j}$ entre el punto de origen $i$ y el punto de destino $j$ y $\mathrm{X}_{i j}$ la variable binaria que define si el vehículo parte del origen $i$ hacia el destino $j$ utilizando el arco $(i, j)$.

$$
\text { minimizar } \sum_{i \in V} D_{i j} X_{i j}
$$

El modelo propuesto incluye la variable Yij que representa la carga con la que el vehículo viaja desde el punto de origen $i$ hacia el punto de destino $j$. La variable con índices invertidos $Y_{j i}$ calcula la capacidad disponible o no utilizada del vehículo de transporte cuando recorre el arco que se origina en el punto $i$ con destino hacia el punto $j$. La primera restricción (1) permite establecer que, si un vehículo pasa por un punto de entrega, entonces dejará disponible una capacidad equivalente a la carga entregada luego de pasar por este.

$$
\sum_{j \in V}\left(Y_{i j}-Y_{j i}\right)=2 q_{i} \forall i \in V \backslash\{0\}
$$

Se incluyó una restricción (2) que establece que el vehículo inicia el transporte en el punto de partida con capacidad utilizada igual a la carga total por entregar a todos los puntos. Se asume que el vehículo tiene capacidad suficiente para llevar toda la carga pendiente de entrega.

$$
\sum_{j \in V \backslash\{0\}} Y_{0 j}=\sum_{i \in V \backslash\{0\}} q_{i}
$$

En coherencia con la condición de carga en el punto de partida se indica que la carga disponible o no utilizada del vehículo en el punto de partida se obtiene de la capacidad C disponible menos la capacidad Yij utilizada en el punto de partida (3).

$$
\sum_{j \in V \backslash\{0\}} Y_{j 0}=C-\sum_{i \in V \backslash\{0\}} q_{i}
$$


El vehículo debe retornar al punto de partida luego de realizar todas las entregas, por lo que la capacidad disponible es igual a $C$ en ese tramo (4).

$$
\sum_{j \in V \backslash\{0\}} Y_{n+1, j}=C
$$

La capacidad utilizada y la capacidad disponible del mismo vehículo solo serán calculadas cuando este transite en el tramo comprendido por el arco $(i, j)$ que une los puntos de entrega (5).

$$
Y_{i j}+Y_{j i}=C X_{i j} \quad \forall(i, j) \in V \times V / i \neq j(5)
$$

Existe la restricción para cada punto que, si un vehículo pasa por un punto $k$, debe llegar a este una sola vez y partir del mismo una única vez (6).

$$
\sum_{i<k} X_{i k}+\sum_{j>k} X_{k j}=2 \quad \forall k \in V \backslash\{0\}(6)
$$

\section{Implementación experimental}

Fueron construidos escenarios considerando dos factores: la cantidad de puntos y la dispersión de los puntos. Para la cantidad de puntos por los que debe pasa el vehículo se consideraron incrementos de 10 puntos, evaluando los valores desde 10 hasta 60 ubicaciones diferentes. Cuando se hallaron valores con cambios significativos en el tiempo de solución se realizaron incrementos de un punto, para mejorar la sensibilidad de la medición.

Los puntos fueron generados mediante números aleatorios que seguían una distribución uniforme. La matriz $D_{i j}$ utilizada fue simétrica, asumiendo que la distancia entre dos puntos de entrega es idéntica en ambos sentidos. Se incluyeron tres escenarios de ubicación de puntos de entrega; se utilizó correlación baja, moderada y alta (Landero y González, 2011), que representan tres casos típicos: tránsito por toda la ciudad (dispersos, $r \approx 0,2$ ), concentración en zonas comerciales (poco dispersos, $r \approx 0,5$ ), tránsito por carretera (puntos concentrados a lo largo de un camino, $r \approx 0,9$ ). En la figura 1 se presenta la muestra para los tres escenarios con 40 puntos. 

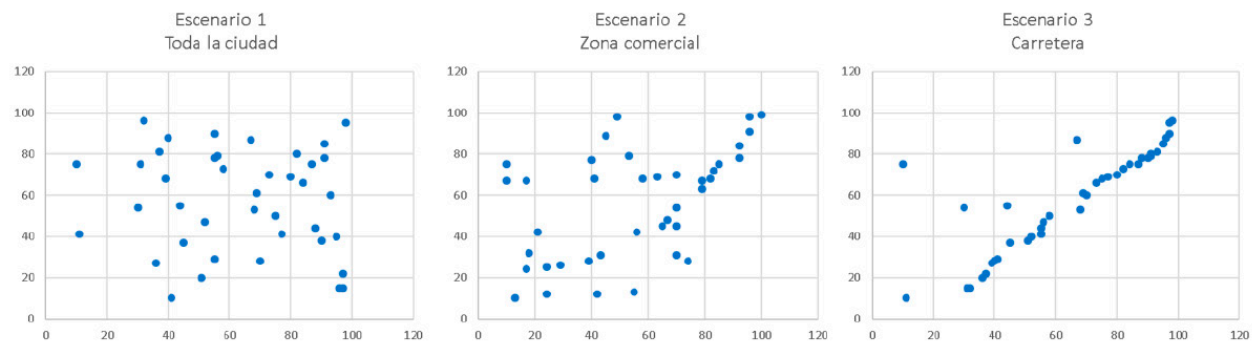

Figura 1. Muestra para 40 puntos

Elaboración propia

Como se mencionó anteriormente, se utilizó un modelo simplificado para encontrar la ruta óptima y se asumieron en todos los escenarios cargas idénticas $q_{i}$ para todos los pedidos con valor igual $a \frac{C}{n}$. La capacidad utilizada para todos los escenarios fue $C_{0}=50$. Se utilizó Lingo 17 para resolver el problema y evaluar los diferentes escenarios creados. Las corridas de los modelos fueron efectuadas utilizando un CPU con procesador Core i5 de Intel con 8 Gb de memoria y sistema operativo Windows de 64 bits.

\section{Resultados}

Los resultados incluyen tiempo y cantidad de iteraciones utilizadas en los escenarios propuestos. La tabla 1 muestra los datos obtenidos para el escenario Toda la ciudad, que reportó los menores tiempos de solución en la mayor cantidad de escenarios evaluados.

Tabla 1. Iteraciones y tiempo de solución para el escenario Toda la ciudad

\begin{tabular}{cccc}
\hline Puntos & Iteraciones & Tiempo (s) & Tiempo (hh:mm:ss) \\
\hline 10 & 557 & 0,19 & $0: 00: 00$ \\
20 & 2887 & 0,95 & $0: 00: 01$ \\
30 & 25714 & 5,86 & $0: 00: 06$ \\
40 & 15529 & 3.81 & $0: 00: 04$ \\
45 & 68181902 & 15994,63 & $4: 26: 35$ \\
50 & 451552143 & 398915,99 & $110: 48: 36$ \\
60 & 365755059 & 96871,11 & $26: 54: 31$ \\
\hline
\end{tabular}

Elaboración propia 


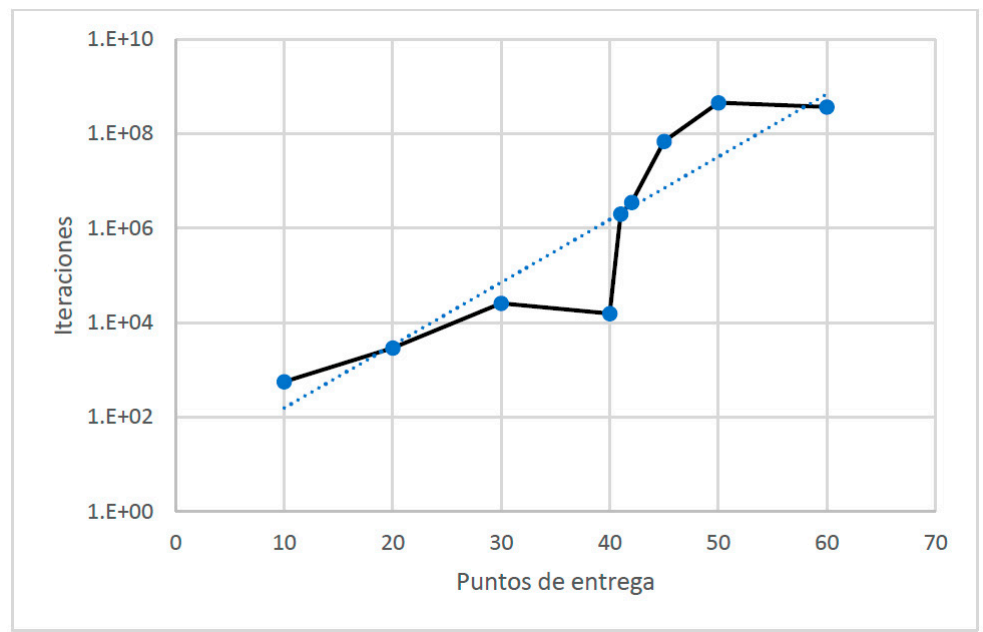

Figura 2. Iteraciones del escenario Toda la ciudad

Elaboración propia

La cantidad de iteraciones necesarias para encontrar la solución en cada uno de los tres escenarios evaluados muestra un crecimiento exponencial. Cuando los puntos se encuentran más cercanos (menor dispersión), el número de iteraciones necesarias para encontrar la solución óptima aumenta y, como consecuencia, el tiempo necesario también se incrementa. El escenario que reportó menor tiempo fue Toda la ciudad, que se caracterizó con puntos que presentaban $r \approx 0,2$. La figura 3 ilustra la cantidad de iteraciones y permite su comparación. En la tabla 2 se muestra el orden de magnitud de las iteraciones necesarias.

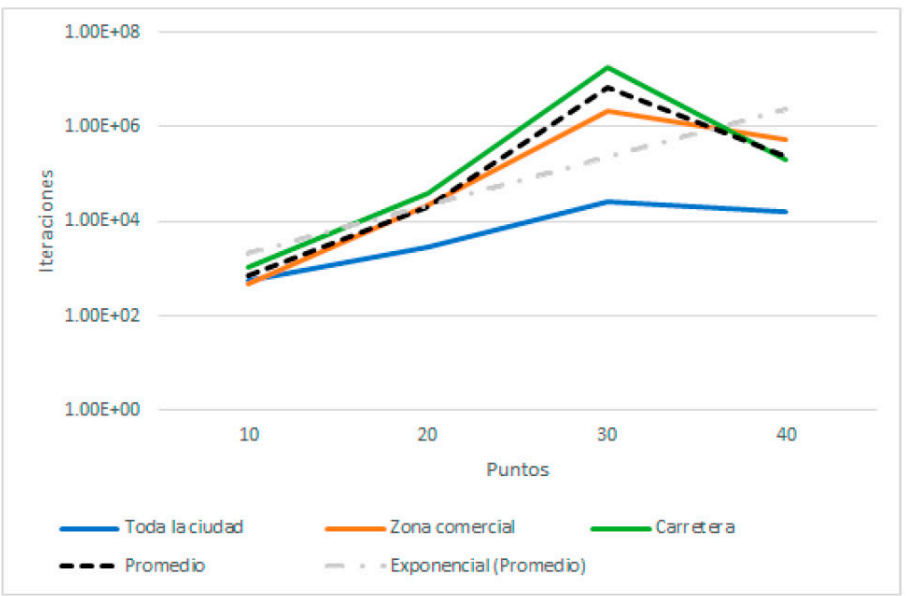

Figura 3. Comparación de la cantidad iteraciones para tres escenarios Elaboración propia 
Tabla 2. Orden de magnitud de las iteraciones necesarias para completar la solución

\begin{tabular}{cc}
\hline Puntos & Om(i) \\
\hline 10 & 3 \\
20 & 3 \\
30 & 4 \\
40 & 4 \\
45 & 8 \\
50 & 9 \\
60 & 9 \\
\hline Elaboración propia &
\end{tabular}

Se encontró una relación lineal directa y significativa $(r=0,891, p<=0,01)$ entre el número de iteraciones y el tiempo en segundos empleado por Lingo 17 para encontrar la solución óptima del modelo propuesto. La figura 4 muestra esta relación lineal.

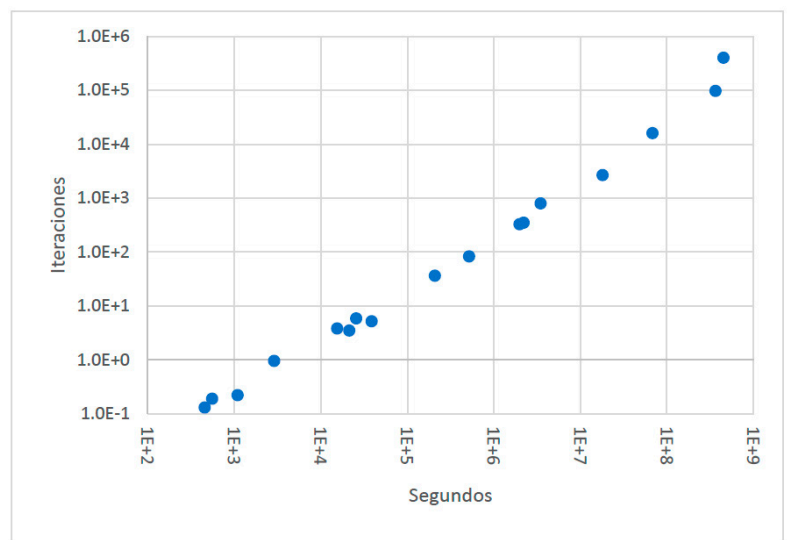

Figura 4. Cuenta de iteraciones vs tiempo de solución Elaboración propia

Mediante regresión se puede determinar el orden de magnitud de las iteraciones y el tiempo aproximado de solución en segundos (s), en función de la cantidad $n$ de puntos que componen la ruta, incluido el punto de partida a donde retornará la unidad una vez que haya concluido las entregas. La ecuación de predicción es $\mathrm{s}=0,002 e^{0,3072 n}$ con un valor de $\mathrm{R}^{2}$ para la regresión de 0,796 , por lo que la complejidad será exponencial $\left(\mathrm{O}\left(\mathrm{k}^{\mathrm{n}}\right)\right)$.

Para estimar el orden de magnitud de las iteraciones se puede calcular la expresión $\mathrm{o}=\log \left(7,268 e^{0,3062 n}\right)$ obtenida por regresión con valor $\mathrm{R}^{2}=0,829$. 


\section{Discusión de resultados}

Los resultados muestran el crecimiento exponencial $\left(R^{2}=0,829\right)$ del número de iteraciones necesarias para encontrar la solución óptima de modelo matemático con variables mixtas y por tanto una complejidad exponencial $\left(\mathrm{O}\left(\mathrm{k}^{\mathrm{n}}\right)\right)$. Este crecimiento es congruente con el número de vértices que evalúa el método simplex y la posterior aplicación del método de bifurcación y acotamiento necesario para determinar el valor de las variables binarias.

La relación directa entre el tiempo de solución y el número de iteraciones evidencia un buen manejo de Lingo 17 de la complejidad debido a la administración en memoria de matrices poco densas que aumentaron de orden en diversos escenarios en los que los puntos de entrega se incrementaron. Esta relación lineal $(r=0,93)$ evidencia que el tamaño matricial no añadió mayor complejidad al tiempo de solución.

Respecto a los resultados de interés para la implementación, se debe tomar en cuenta que las unidades de transporte son asignadas a conductores que normalmente laboran en turnos de ocho horas por regulaciones laborales y fatiga. Cuando existen múltiples conductores en la misma unidad, la jornada laboral puede ampliarse a 10 o 12 horas (Ministerio de Transportes y Comunicaciones, 2009). Esta condición determina el tiempo máximo disponible para la asignación de la ruta a un vehículo de transporte destinado a la entrega de mercancías. En el caso de que el programador de rutas necesite realizar ajustes o reasignaciones, el tiempo se reducirá a la mitad o a la tercera parte. Con estas limitaciones, los resultados muestran que con el modelo utilizado es posible asignar rutas hasta un máximo entre 40 y 50 puntos con el tiempo disponible de hasta ocho horas. Estos resultados son similares a los de Laporte (2009) que informó obtener soluciones hasta 60 puntos para el modelo original hasta con 5 vehículos, sin indicar el tiempo utilizado.

La complejidad de un modelo matemático está expresada por la cantidad de restricciones del modelo y la cantidad de variables discretas incluidas. En el modelo de prueba la cantidad de restricciones corresponde a $(n+1)^{2}+n$. por lo que tiene una complejidad $O\left(n^{2}\right)$ y el $50 \%$ de las variables son binarias. En función de los tiempos de solución obtenidos durante las pruebas, al utilizar los criterios de selección de tiempos razonables expuestos, se determinó que un orden de magnitud inferior a siete para la cantidad de iteraciones resulta práctico y viable para implementarse en soluciones informáticas basadas en este modelo. Estos resultados permiten inferir que el rango al que pertenece el máximo de puntos será menor cuando se utilicen otros modelos de complejidad similar o mayor a $\mathrm{O}\left(\mathrm{n}^{2}\right)$ y que consideren las limitaciones reales de asignar cargas a múltiples vehículos, además de disponer de capacidad limitada.

Al revisar los tiempos de solución reportados por Daza, Montoya y Narducci (2009) con soluciones heurísticas y metaheurísticas, sería recomendable la evaluación de estas técnicas para la resolución del VRP fuera del rango propuesto. 
Contrariamente a lo que el sentido común indica, cuando los puntos están dispuestos con una mayor correlación, el tiempo de solución es mayor, pues es necesaria una mayor cantidad de iteraciones para encontrar la solución óptima. Este resultado puede deberse a que cuando estos puntos se encuentran dispuestos así, las diferencias entre distancias son mucho menores y puede resultar más difícil para el algoritmo seleccionar una solución que sea mejor a las otras y por tanto puede que exista una cantidad mayor de cambios en la solución hasta encontrar la solución óptima. Por el contrario, cuando los puntos se encuentran dispersos la solución óptima puede ser encontrada antes, al existir mayores diferencias que permiten descartar con menos iteraciones las otras soluciones.

La importancia de alinear las operaciones de reposición con los clientes fue reportada por la consultora Booz Allen Hamilton que las denominó empresas "personalizadoras inteligentes", encontrando que exhibieron una brecha de rendimiento de 2:1 sobre aquellas compañías que no lo hicieron (Gattorna, 2009). Los resultados muestran que no es viable la optimización lineal para grandes cantidades de puntos de entrega, por ello la agrupación de estos por criterios que permitan una previa agrupación basada en una segmentación alineada con las operaciones de reparto generaría no solo una reducción de las probabilidades de error humano, una mayor eficiencia y menor costo en el uso de transporte, sino un mayor rendimiento que incremente directamente el nivel de servicio y la satisfacción del cliente.

\section{Conclusiones y recomendaciones}

A partir de este estudio experimental se concluye:

- La solución del VRP presenta una complejidad exponencial $(\mathrm{O}(\mathrm{kn}))$ a medida que los puntos de entrega se incrementan debido al tiempo y las iteraciones necesarias para resolver el modelo de programación lineal. Además, la relación entre el tiempo de solución en segundos y la cantidad de iteraciones necesarias del solver Lingo 17 es lineal, sin haberse encontrado diferencias significativas debido a los cambios en el orden de la matriz básica.

- Con la potencia computacional actual la resolución de problemas de programación lineal mixta con este tipo de formulación permite optimizar empleando tiempos razonables y alcanzar una solución con magnitud de millones o decenas de millones de iteraciones.

- Basados en la cantidad de puntos de entrega es posible estimar el orden de magnitud de la cantidad de iteraciones y el tiempo requerido para encontrar la solución óptima.

Se recomienda un rango de 40 a 50 puntos de entrega como máximo para modelos de complejidad $\mathrm{O}\left(\mathrm{n}^{2}\right)$ con variables binarias, dentro del cual el tiempo de solución aplicando programación lineal en el VRP es razonable en el contexto del desarrollo de un sistema de 
soporte para la toma de decisiones. Fuera de ese rango es conveniente evaluar algoritmos heurísticos o metaheurísticos que brinden soluciones no óptimas, pero en tiempos razonables. Los tiempos en escenarios dispersos (toda la ciudad) son menores que en escenarios concentrados (carretera). Este hallazgo es una motivación para ampliar la investigación ya que en este tipo de escenarios la solución puede ser obvia para el personal a cargo mientras que la computadora tardará más en proporcionar una solución óptima.

\section{Otras implicancias del estudio}

Desde el enfoque de gestión:

- La viabilidad de encontrar soluciones óptimas para las rutas de vehículos en tiempo razonable determina que la optimización mediante programación lineal con modelos de complejidad cuadrática evalúe como máximo valores cercanos a los 50 puntos de entrega.

- Si se requiere considerar en el modelo variables como carga o inventario disponible, o valorización de pedidos, esto hará que la complejidad aumente y los puntos de entrega máximos sean menores.

- La complejidad determina que se debe realizar una asignación previa de puntos de entrega a las unidades de transporte por criterios que pueden ser diferentes a la distancia entre puntos cuando es necesario programar rutas para una mayor cantidad de puntos. La elección de criterios tácticos adecuados puede impactar significativamente en la eficiencia y productividad de la empresa, por lo que deja un margen para la discusión de los criterios clave a considerar por parte de los tomadores de decisiones y responsables del establecimiento de políticas correctas. La segmentación de los clientes puede ser uno de los criterios clave, pues las evidencias muestran rendimientos mayores en las empresas que han realizado personalización de la entrega como medio para mejorar su relación con el cliente.

- El reemplazo del trabajo manual aún no es viable del todo, pues existen escenarios evidentes para la experiencia humana como el de puntos de reparto poco dispersos en los que se requiere personal experimentado y capacitado que tome decisiones, dejando la resolución de escenarios complejos como puntos altamente dispersos con el uso de software, para agilizar los procesos de distribución de productos.

Desde el enfoque de investigación:

- La necesidad de realizar asignaciones previas para problemas de gran envergadura conduce a la evaluación de propuestas de resolución mediante programación por metas cuando coexistan múltiples criterios para realizar la asignación previa. 
- La evaluación del impacto del uso de múltiples unidades de características diversas puede determinar rangos diferentes para la aplicación de las técnicas de optimización.

Es necesario conocer otros factores distintos a la distancia o costo de entrega que orienten la decisión en la elección de criterios a tomar en cuenta para priorización de pedidos pendientes de atención.

\section{Referencias}

Alvarez, P., Lerga, I., Serrano, A. y Faulin, J. (2017). Considering Congestion Costs and Driver Behaviour into Route Optimisation Algorithms in Smart Cities (pp. 39-50). DOI:10.1007/978-3-319-59513-9_5.

Arboleda-Zúñiga, J., López, A. X. y Lozano, Y. L. (2016). El problema de ruteo de vehículos [VRP] y su aplicación en medianas empresas colombianas. Ingenium, 10(27), 29-36.

Azzara, C. V. (2010). Questionnaire Design for Business Research: Beyond Linear Thinking-an Interactive Approach. Mustang: Tate Publishing \& Enterprises.

Baldacci, R., Hadjiconstantinou, E. y Mingozzi, A. (2004). An exact algorithm for the vehicle routing problem based on the set partitioning formulation with additional cuts. Operations Research, 52(5), 723-738. DOI:10.1287/opre.1040. 0111.

Cook, S. A. (1983). An overview of computational complexity. Communications of the ACM (Vol. 26).

Dantzig, G. B. (2002). Linear Programming. Operations Research, 50(1), 42-47. DOI:10.1287/ opre.50.1.42.17798.

Daza, J. M., Montoya, J. R. y Narducci, F. (2009). Resolución de problema de enrutamiento de vehículos con limitaciones de capacidad utilizando un procedimiento metaheurístico de dos fases. Eia, 1(12), 23-38.

Gattorna, J. (2009). Cadena de abastecimientos dinámicas: Cómo movilizar la empresa alrededor de lo que los clientes quieren (1 ${ }^{\mathrm{a}}$. ed.). Bogotá: Ecoe Ediciones.

Golden, B. L., Wasil, E. A., Kelly, J. P. y Chao, I.-M. (1998). The impact of metaheuristics on solving the vehicle routing problem: Algorithms, problem sets, and computational results. En Fleet Management and Logistics (pp. 33-56). Boston, MA: Springer US. DOI:10.1007/978-1-4615-5755-5_2.

Han, H. y Cueto, E. P. (2015). Waste Collection Vehicle Routing Problem: A Literature Review. PROMET - Traffic \& Transportation, 27(4), 345-358. https://doi.org/10.7307/ptt.v27i4.1616. 
Landero, R. y González, M. (2011). Estadística con SPSS y metodología de la investigación (1a. ed.). México: Trillas.

Laporte, G. (2009). Fifty Years of Vehicle Routing. Transportation Science, 43(4), 408-416. DOI:10.1287/trsc.1090.0301

Limeños pierden el 25 \% de sus ingresos por el tránsito vehicular. (2017). La República. Recuperado de: http://larepublica.pe/economia/865633-limenos-pierden-el-25-de-sus-ingresos-porel-transito-vehicular

Lovelock, C. y Wirtz, J. (2009). Marketing de servicios (6ª. ed.). México: Pearson Educación.

Machuca, J. y Taquía, J. (2009). Balanza comercial de los combustibles líquidos derivados del petróleo mediante dinámica de sistemas y simulación. Ingeniería Industrial, 27, 61-79.

Onut, S., Kamber, M. R. y Altay, G. (2014). A heterogeneous fleet vehicle routing model for solving the LPG distribution problem: A case study. Journal of Physics: Conference Series, 490. DOI:10.1088/1742-6596/490/1/012043.

Ministerio de Transportes y Comunicaciones (2009). Decreto Supremo №. 017-2009-MTC.

Srivatsa Srinivas, S. y Gajanand, M. S. (2017). Vehicle routing problem and driver behaviour: a review and framework for analysis. Transport Reviews, 37(5), 590-611. DOI:10.1080/0144 1647.2016.1273276.

Tzeng, G.-H., Cheng, H.-J., y Huang, T. D. (2007). Multi-objective optimal planning for designing relief delivery systems. Transportation Research Part E: Logistics and Transportation Review, 43(6), 673-686. DOI:10.1016/j.tre.2006.10.012. 
